\title{
Interactive Wood Combustion for Botanical Tree Models
}

\author{
SÖREN PIRK, Stanford University \\ MICHAŁ JARZĄBEK, Adam Mickiewicz University \\ TORSTEN HÄDRICH, KAUST \\ DOMINIK L. MICHELS, KAUST \\ WOJCIECH PALUBICKI, Adam Mickiewicz University
}
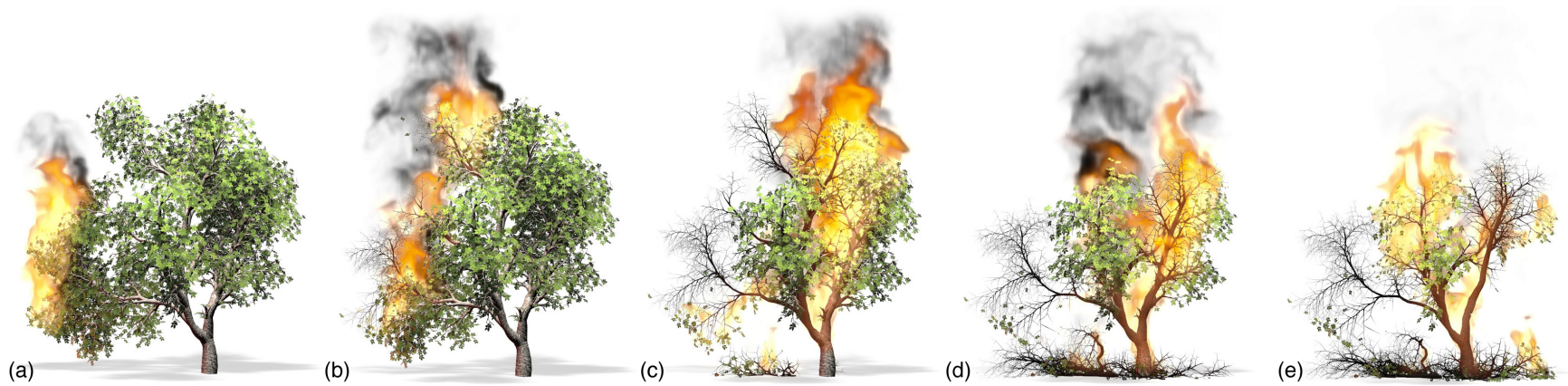

Fig. 1. Combustion of a tree model: a tree is exposed to fire until the branching structure reaches its ignition temperature (a). The combustion releases energy stored in the tree organs and propagates through the entire tree model until it reaches its peak (b). The combustion causes branches to bend and break (c) while the flames conquer more branches (d) and eventually burn the entire tree model (e).

\begin{abstract}
We present a novel method for the combustion of botanical tree models. Tree models are represented as connected particles for the branching structure and a polygonal surface mesh for the combustion. Each particle stores biological and physical attributes that drive the kinetic behavior of a plant and the exothermic reaction of the combustion. Coupled with realistic physics for rods, the particles enable dynamic branch motions. We model material properties, such as moisture and charring behavior, and associate them with individual particles. The combustion is efficiently processed in the surface domain of the tree model on a polygonal mesh. A user can dynamically interact with the model by initiating fires and by inducing stress on branches. The flames realistically propagate through the tree model by consuming the available resources. Our method runs at interactive rates and supports multiple tree instances in parallel. We demonstrate the effectiveness of our approach through numerous examples and evaluate its plausibility against the combustion of real wood samples.
\end{abstract}

CCS Concepts: • Computing methodologies $\rightarrow$ Interactive simulation; Physical simulation;

Additional Key Words and Phrases: Botanical Tree Models, Visual Models of Trees, Natural Phenomena, Interactive Modeling, Wood Combustion

\section{ACM Reference format:}

Sören Pirk, Michał Jarząbek, Torsten Hädrich, Dominik L. Michels, and Wojciech Palubicki. 2017. Interactive Wood Combustion for Botanical Tree Models. ACM Trans. Graph. 36, 4, Article 197 (July 2017), 12 pages. https://doi.org/10.1145/3130800.3130814

Permission to make digital or hard copies of all or part of this work for personal or classroom use is granted without fee provided that copies are not made or distributed for profit or commercial advantage and that copies bear this notice and the full citation on the first page. Copyrights for components of this work owned by others than ACM must be honored. Abstracting with credit is permitted. To copy otherwise, or republish, to post on servers or to redistribute to lists, requires prior specific permission and/or a fee. Request permissions from permissions@acm.org.

(C) 2017 Association for Computing Machinery.

0730-0301/2017/7-ART197 \$15.00

https://doi.org/10.1145/3130800.3130814

\section{INTRODUCTION}

As frequent objects in everyday scenes, models of trees and plants serve as important content in various application domains, ranging from architectural design and urban modeling to computer games and more recently the motion planning of autonomous agents. While many approaches exist to readily model the structure and visual appearance of vegetation, modeling the dynamic behavior of plants and their interaction with the environment still pose challenging problems. Faithfully modeling the plants' response to physical effects, such as wind, rain, or snow arguably play a key role in many situations. However, the complexity of these natural phenomena renders their joint simulation impossible in most processing pipelines. Moreover, artists often require a nuanced means of control to adapt such effects as part of the content creation and the storytelling in their applications.

Traditionally, the adaptivity of trees and plants is modeled by explicitly considering the environment [Měch and Prusinkiewicz 1996], inverse procedural modeling [Stava et al. 2014], modeling the competition for resources [Palubicki et al. 2009], or simulated adaptation [Pirk et al. 2012]. Only recently, efforts in plant modeling concentrate on the dynamic behavior by simulating the physics response of plants. Realistic motions, also including the faithful simulation of growth [Longay et al. 2012], the adaptation to surfaces [Hädrich et al. 2017], and as induced by realistic materials [Wang et al. 2017], play a key role for the convincing behavior of plants.

Current methods in computer graphics for simulating the combustion of solids ignore essential parts of tree combustion and cannot be easily adapted to botanical models of trees [Melek and Keyser 2002]. Physically-based simulations of wood combustion explored in other research areas, such as material sciences [Thi et al. 2016], are computationally demanding and only focus on the combustion 
of wood samples in laboratory conditions. In forestry, where the study of tree combustion is a key topic, no models have been devised at the scale of tree geometry [Seidl et al. 2012].

In this paper we advance tree modeling by introducing a combustion model for trees that is biologically plausible while also being computationally efficient. The key idea to our approach is to jointly simulate tree motion, fire, and the combustion of plant tissue. Fire is simulated through a volumetric grid-based fluid solver that transfers heat to plant tissue and initiates the combustion of the model. Conversely, a burning tree model releases heat to its environment, resulting in a positive feedback loop which leads to fire spread and maintains the combustion (Fig. 1).

A tree is represented by a dual formulation that employs particles for modeling the branching structure and a surface mesh for simulating the combustion. We use a position-based dynamics approach with connected particles to enable dynamic and realistic motions of branches [Kugelstadt and Schömer 2016]. Each particle stores biological and physical attributes to drive the kinetic behavior of the plant and the exothermic reaction of the combustion. Burning tree models results in inhomogeneous transformations that are difficult to simulate jointly with the fluid dynamics of fires. Moreover, changes in the structure and the biomechanical properties of the plants directly affect the combustion.

In summary our key contributions are: (1) we propose a method for the combustion of botanical tree models based on heat transfer and integration of plausible physics of tree models and fluid dynamics; (2) we model trees based on position-based dynamics and Cosserat physics to support realistic plant motions and jointly simulate them with fluid dynamics for fire; (3) our framework maintains animation-ready plants at all stages of the simulation and supports the efficient interaction with plants in real-time; (4) we evaluate our implementation against the combustion of real wood samples.

\section{RELATED WORK}

Early approaches of modeling vegetation focus on the refined definition of branching structures through a variety of approaches, such as fractals and repetitive patterns [Oppenheimer 1986] or L-Systems [Prusinkiewicz 1986]. Later methods focus on the interaction of plants with their environment, through query modules [Měch and Prusinkiewicz 1996], inverse procedural modeling [Stava et al. 2014] and through explicitly modeling the competition for resources [Palubicki et al. 2009]. Data-driven and sketchbased approaches focus on modeling plants from sensor data [Livny et al. 2011; Neubert et al. 2007] or user-defined sketches [Ijiri et al. 2006; Okabe et al. 2007].

Recent approaches provide more nuanced ways to efficiently model branching structures with an emphasis on simulating the response of trees to their environment [Pirk et al. 2014, 2012], interactive growth [Pirk et al. 2012] or FEM simulation [Zhao and Barbič 2013]. Longay et al. [2012] introduce an interactive tree modeling system that jointly models trees based on the competition for resources and user defined sketches. These approaches have in common to lift tree models from static branching structures to more dynamic representations, however, they do not model the interaction of plants with plausible physics.
Another line of work invests in techniques for increasing the realism of the physics response of elastic filaments, such as plant tendrils or assemblies of fibers and nearly one-dimensional slender structures in general [Casati and Bertails-Descoubes 2013]. Selle et al. [2008] simulate the dynamics of thinned out hair with several thousand individual fibers. A single fiber is constructed using a tetrahedral mass-spring system that is furnished with additional ad-hoc springs in order to prevent from a volumetric collapse. Such mass-spring models are rather popular in graphics because of their efficiency, however, physically accurate results are complicated to produce since realistic material parameters are hard to integrate without running into numerical issues [Michels et al. 2015].

Pai [2002] introduce the theory of Cosserat rods to the computer graphics community and it has been shown that this leads to physically accurate results for modeling the dynamics of rods and fibers [Bertails et al. 2006]. More recently, Kugelstadt and Schömer [2016] showed that even bending and torsion of elastic rods can be approximated with a position-based dynamics (PBD) framework [Müller et al. 2007], by introducing additional constraints. Elastic rods play a key role in simulating the physics response of tree models. Hädrich et al. [2017] model the growth behavior and physics response of climbing plants with an emphasis on dynamic control and interactive authoring.

The simulation and animation of fire has a long tradition in computer graphics. Commonly, fire is simulated through grid-based fluid solvers that allow capturing laminar and turbulent features in flames [Hong et al. 2007; Nguyen et al. 2002; Stam 1999] and in smoke [Fedkiw et al. 2001]. Pegoraro and Parker [2006] introduce a method for the physically-based rendering of fire dynamics that builds on molecular physics and Horvath and Geiger [2009] propose an efficient implementation that combines a coarse particle simulation with high resolution view-dependent grids.

The combustion of rigid bodies can be modeled through volumetric grids that allow to track disconnected propagating fronts [Liu et al. 2012; Melek and Keyser 2005; Zhao et al. 2003] and to spread fires on surfaces [Chiba et al. 1994]. Hong et al. [2010] propose a method to model fires under general geometric constraints that even allows to burn plant models. Finally, Stomakhin et al. [2014] introduce a point-based technique for melting and solidifying materials through simulating heat transfer. Their method allows to capture the thermodynamic properties in different materials and to alter their mechanical properties, however, it does not support interactive modeling and heat diffusion in real-time.

Research in forestry and botany focuses on the heat transfer of entire stands of trees [Bohren and Thorud 1973; Encinas et al. 2007], on models to describe the biomechanical properties of wood with respect to charring behavior [Lizhong et al. 2002], fire resistance of wood [Lawes et al. 2011], or the pyrolysis of trees and plants in general [Demirbaş 2000]. The resistance of trees against fires is mostly determined by the thickness of their bark and several approaches try to quantify the resistance behavior of certain species [Lawes et al. 2011]. Many of these techniques describe accurate models of fire propagation and heat transfer in plants, however, they cannot directly be applied to detailed geometric models of trees and do not support the interactive exploration of combustion in real-time. 


\section{OVERVIEW}

The realistic combustion of tree models requires simulating fluid dynamics for fire, modeling dynamic tree models - including geometric deformations of the branch mesh, and the simulation of the heat transfer. Fig. 2 shows an overview of our system. The input to our method is a tree model represented as a skeletal graph, and a set of parameters for both, the fire simulation and the combustion.

Fire is simulated through a grid-based fluid solver that approximates the Navier-Stokes equations. For the implementation we rely on established solvers that allow to simulate characteristic features of flames at interactive rates [Fedkiw et al. 2001]. The combustion of flames is simulated based on an approach similar to Melek and Keyser et al. [2002], that supports laminar and turbulent flames as well as the burning of solid and gaseous fuels.

Trees are modeled as environmental-sensitive particles that allow plausible branch motions. Branches are formed by connecting strings of particles in a hierarchical relationship. We couple the particles with Cosserat physics [Kugelstadt and Schömer 2016], which enables more realistic motions for solitary trees, including the bending and torsion of branches. Moreover, each tree particle is associated with physical and biological attributes, such as strain, moisture, and insulation, that define the models' response to the fire simulation.

The branch mesh is dynamically updated according to attribute changes of the particles and the combustion simulation. Our method propagates heat on the surface of branches and deforms the branch geometry according to the combustion. The combustion starts when plant tissue is exposed to a fire until the ignition temperature is reached. The temperature propagates through the plant tissue depending on the local material properties.

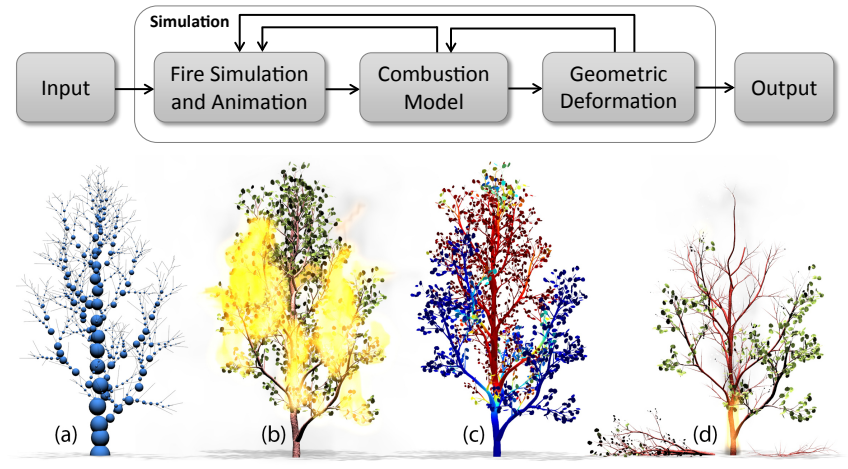

Fig. 2. Overview: a tree model is converted into a set of connected particles (a); we simulate fires (b) and couple them with a biologically-plausible combustion model for botanical tree models (colors indicate temperature) (c) that causes complex geometric deformations (d). Our framework runs at interactive rates and maintains animation-ready plants (output).

\section{DYNAMIC TREE MODELS}

Dynamic motions are essential for the realistic simulation of combustion. While temperature differences of flames and surrounding air produce wind that sets branches and leaves in motion, the combustion of plant tissue gradually reduces the thickness of branches and causes them to bend, break, and eventually fall down on the ground. Our system is able to efficiently simulate these effects by exploiting a particle-based representation for trees and by using simple algebraic expressions for the integration of position and orientation updates for branching structures.

Similar to other approaches [Livny et al. 2011], the input to our method is a graph-based representation of a tree model. The branching structure is defined as a skeletal graph $G=\{N, H\}$, where $N$ are the nodes and $H$ the edges. Each edge connects two nodes $n_{\mathrm{s}}$ and $n_{\mathrm{t}}$ to produce a hierarchal and non-cyclic relationship, where $n_{s}$ is the parent of $n_{\mathrm{t}}$. One node $n_{\text {root }}$ is the root node of the tree model. Single branches are defined as chains of edges $C=\left\{h_{1}, h_{2}, \ldots, h_{n}\right\}$, where $n$ denotes the length of a given branch.

For the simulation of trees we use a discretized Cosserat rod tree representation. Cosserat rods are described by a smooth curve $\mathbf{r}(s):\left[s_{0}, s_{1}\right] \rightarrow \mathbb{R}^{3}$. Each point on the curve has an orthonormal material frame with the attached basis vectors $\left\{\mathbf{d}_{1}(s), \mathbf{d}_{2}(s), \mathbf{d}_{3}(s)\right\}$. The material frame vectors $\mathbf{d}_{k}$ are called directors. The orthonormal frame can be described by a quaternion as a rotation of the world space basis vectors $\left\{\mathbf{e}_{1}, \mathbf{e}_{2}, \mathbf{e}_{3}\right\}$ (Fig. 3 , left). The rods are discretized by a set of particles and material frames between them. Two particles and one quaternion that stores the orientation of the material frame will be called rod element (Fig. 3, right). Adjacent rod elements share one particle.
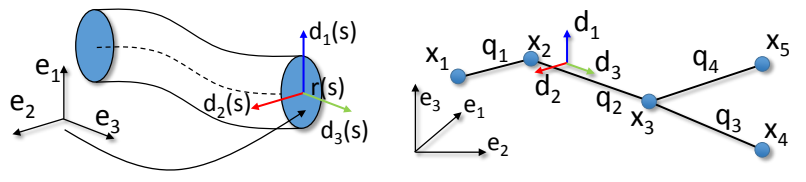

Fig. 3. The geometric configuration of a discretized rod. The vectors $\mathbf{e}_{k}$ denote the world space basis coordinates and the directors $\mathbf{d}_{k}$ the local material frame of the rod.

We convert the graph-based representation into rod elements by recursively traversing the tree graph starting at $n_{\text {root }}$. Nodes in the tree graph are represented by particles and edges are converted into rod elements. We compute the quaternion of a rod element by rotating the quaternion $u_{n-1}$ of the parent rod element so that the director $\mathbf{d}_{3_{n-1}}$ aligns with the current edge direction:

$$
u_{n}=R\left(\mathbf{d}_{3_{n-1}} \times \hat{\mathbf{h}}_{n}, \angle\left(\mathbf{h}_{n}, \mathbf{h}_{n-1}\right)\right) u_{n-1},
$$

where $u_{n}$ is the quaternion for the new rod element, $u_{n-1}$ the orientation of the previous rod element, $R$ returns the rotation quaternion for a given axis and angle, and $\mathbf{d}_{3_{n-1}}$ is the director of the previous rod element, $\hat{\mathbf{h}}_{n}$ the unit vector of the new edge, $\mathbf{h}_{n-1}$ the edge of the parent rod element, and $\angle$ denotes the angle between two vectors. The length of the rod element is given by the edge length from the tree graph. We associate the thickness of a graph node with the corresponding particle for generating the surface mesh.

Tree dynamics are realized by employing position and orientation based Cosserat rods [Kugelstadt and Schömer 2016] using connected particles. Their method accounts for bending and torsion of elastic rods, which makes it well suited for the dynamic modeling of branching structures. In contrast to the original approach, we additionally define branching structures. Each particle can have 
multiple outgoing rod elements to its children (Fig. 3, right) and each child rod element stores an orientation and the corresponding length. Cosserat physics can be applied to branching structures by summing up the corrections for particle positions and rod element orientations in a first step followed by the application of these changes to the particles and rod elements respectively.

\section{FIRE SIMULATION}

Colloquially, fire denotes a set of flames and its evolution during the process of combustion. More precisely, fire can be defined as chemical reaction of oxygen and flammable gases (fuel) that releases various reaction products like water and carbon dioxide, as well as heat and light, which together poses the fire.

We simulate fire as a temperature field evolving in a gaseous fluid according to Fedkiw et al. [2001]. After the velocity update, the temperature is updated according to a modified diffusion process. For that, the distribution of the temperature $T$ in the fluid domain is governed by

$$
\partial_{t} T+\vec{u} \cdot \nabla T=\alpha \nabla^{2} T+\gamma\left(T-T_{\mathrm{amb}}\right)^{4}+s_{\mathrm{T}},
$$

where $\vec{u}$ denotes the fluid's velocity field, $T_{\mathrm{amb}}$ the ambient temperature, and $\alpha$ the thermal diffusivity of air. The term involving the fourth power of the temperature difference and a non-negative constant $\gamma$ is the radiative cooling term adapted from Nguyen et al. [2002]. The time- and position-dependent function $s_{\mathrm{T}}$ represents the input of heat generated by the burning process into the fire simulation. It is provided by the combustion simulation. Furthermore, the combustion process generates smoke. Its density $S$ is updated according to a passive advection process with the fluid described by

$$
\partial_{t} S+\vec{u} \cdot \nabla S=s_{S} .
$$

The source term $s_{S}$ is also provided by the combustion model. Both, $s_{\mathrm{T}}$ and $s_{\mathrm{S}}$ are described in Sec. 6.2.

The fluid equations are discretized on a rectangular Eulerian grid using finite differences. We use Lie operator splitting to numerically solve equations with multiple terms. All advection terms are handled by the unconditionally stable version of the MacCormack method [1969], while the projection of the tentative velocity is performed using a Jacobi iteration. Velocity damping and temperature cooling terms are integrated analytically. All remaining terms use forward Euler integration. We do not use trees as a boundary condition for the fluid simulation, but instead use the heat and smoke generation terms $s_{T}$ (Eq. 2) and $s_{S}$, (Eq. 3), provided by our combustion model. Furthermore, we define the outer boundary of our fluid domain as $p=0, t=T_{\mathrm{amb}}, S=0$ and $\partial_{n} \vec{u}=0$ and do not explicitly model the ground boundary.

\section{COMBUSTION OF TREE MODELS}

The combustion of a solid fuel starts when it is exposed to heat until its ignition temperature is reached. For charring materials, such as wood, cloth, and paper, combustion additionally involves the decomposition of material into char and flammable gases (fuel). This process, which can be considered as a part of the combustion, is called pyrolysis [Emmons and Atreya 1982]. At a high level of abstraction the phenomenon can be divided into three separable components: (1) thermal decomposition of material (pyrolysis); (2) conditions for flame spread (ignition); (3) heat and mass transfer from the burning zone into the plant tissue (fire spread). For our method, we assume pyrolysis to occur in a relatively thin zone at the branch tissue, instead of operating on the full volume of a branch. This propagating zone is referred to as the pyrolyzing front. In the following sections we discuss a mathematical model that includes these components and allows for modeling the realistic combustion of woody tissue for botanical tree models.

\subsection{Interactive Wood Combustion}

We aim to create a computationally efficient model for the combustion of wood that can be used for three-dimensional models of trees. Specifically, our model expresses the pyrolysis of wood, including the evaporation of moisture, the formation of a char layer, and the reduction of virgin wood. Moreover, we model the ignition of woody tissue and the subsequent flame spread including the heat transfer across the tree as well as in the surrounding air.

Our combustion model relies on a discrete representation of the branch geometry. We use a polygonal surface mesh, where each surface element (triangle) represents a volumetric portion of the branch, which can be defined as a set of points traced out by a continuous projection of each surface point onto the closest point on the branch axis (Fig. 4, left and inset, right). Each surface element is associated with information about the total mass $M$ of the underlying branch volume, the surface temperature at this location $T_{S}$, the water content $W$, the thickness of a possible char layer $H_{\mathrm{C}}$, the thickness of the virgin wood $H$ and the area of the inwardly moving, pyrolyzing front $A$. Spatial transfer of informa-

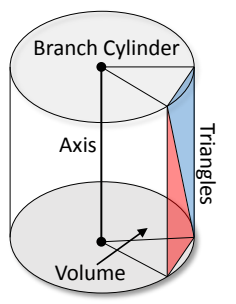

tion happens only in terms of heat in our model. This means, we neglect the spatial mass transfer, such as the release of flammable gases, and assume an immediate effect of pyrolysis on e.g. air temperature. Given this scale of abstraction we can summarize the most important assumptions in our combustion model as follows:

- The tissue layer currently undergoing pyrolysis is approximated by a surface (Pyrolyzing Front).

- The thickness of the char layer is determined by the amount of pyrolyzed wood (Char Contraction).

- The char layer has an insulating effect proportional to its thickness (Char Insulation).

- The rate of wood combustion is proportional to surface temperature (Reaction Rate Coefficient).

- Heat transfer from air to surface is inversely proportional to the amount of water content of surface elements (Moisture).

- Pyrolysis adds heat to the surrounding air proportional to the mass loss rate of surface elements (Heat Transfer).

6.1.1 Pyrolyzing Front. Pyrolysis occurs in a thin layer of the branch tissue. We assume a fixed temperature for pyrolysis and a single step global reaction scheme (infinite rate kinetics) - the virgin wood is decomposed into volatiles and char [Galgano and Di Blasi 2005]:

$$
\text { Wood }+ \text { Heat } \rightarrow \text { Fuel }+ \text { Char }
$$




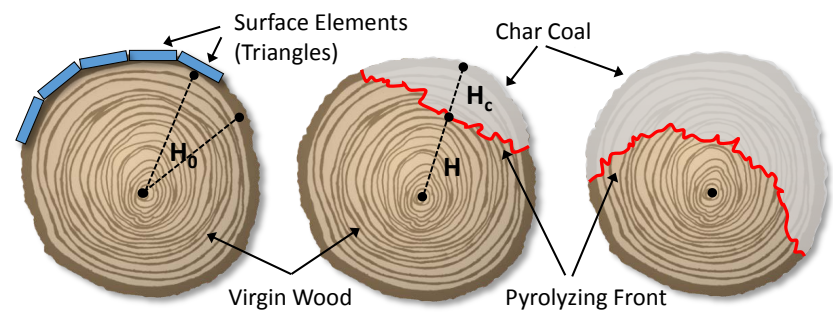

Fig. 4. The cross section of a branch: branches are discretized as triangular surface mesh (left). We define pyrolysis as propagating front that transforms virgin wood into char coal (middle). As pyrolysis continues, the pyrolyzing front moves towards the branch axis (right).

We represent the pyrolyzing front as a two-dimensional triangular surface with area $A$, which is given by $A=A_{0} H / H_{0}$. The original depth of the volumetric portion represented by a surface element is denoted by $H_{0}$ and the current height of the pyrolyzing front is given by $H$. At the onset of pyrolysis the pyrolyzing front is located at the branch surface and matches exactly the area $A_{0}$ of the surface element $\left(H=H_{0}\right)$. As pyrolysis continues, the pyrolyzing front will move from the surface towards the branch axis (the value of $H$ decreases), resulting in a decreasing area $A$ (Fig. 4 , middle and right). The current height $H$ of the pyrolyzing front is given by

$$
H=\sqrt{2 \frac{M}{\rho} \frac{H_{0}}{A_{0}}},
$$

where $\rho$ is the density of the virgin wood. $M$ denotes the current mass of virgin wood expressed as a function of time. Thus, the current height $H$ of the pyrolyzing front in each time step of the simulation can be obtained from the value of the current mass of virgin wood $M$, as the remaining factors in Eq. (4) are constant.

6.1.2 Char Contraction Factor. As the pyrolyzing front is moving towards the branch center in our model, we calculate the formation of a char layer. Studies in wood engineering show that a reasonable approximation for the char layer thickness can be obtained from the current height of the pyrolyzing front $H$. We use the char contraction factor $\kappa$ to obtain an estimate for the char layer thickness $H_{\mathrm{c}}$. The char contraction factor is defined as the fraction of the thickness of the char layer, when the pyrolysis is complete, and the original thickness of the virgin wood. Various species of trees and types of wood can have different char contraction factors [Parker 1989] We use an experimentally derived char contraction factor $\kappa$, in the range of $[0.5,1.0]$, to calculate the char layer thickness

$$
H_{\mathrm{c}}=\kappa\left(H_{0}-H\right) \text {. }
$$

6.1.3 Char Insulation. Various experiments in wood engineering indicate that, with the formation of a char layer, the mass loss rate of virgin wood starts decreasing significantly [Friquin 2011]. Therefore, the insulation effect of char is an important phenomenon to include in a combustion model of wood. However, a detailed physical simulation of this phenomenon would necessitate a description of volumetric heat transfer across the char layer and different woody tissues. We therefore approximate the insulating effect of the char layer with the non-dimensional parameter $c$, which is non-linearly proportional to the char layer thickness $H_{\mathrm{c}}$. It is given by

$$
c=c_{\min }+\left(1-c_{\min }\right) e^{-c_{\mathrm{r}} H_{\mathrm{c}}} \text {. }
$$

At the start of pyrolysis, $c$ equals 1 as the char layer thickness $H_{\mathrm{c}}$ is equal to 0 . Subsequently, as $H_{\mathrm{c}}$ increases, the char insulation parameter $c$ decreases exponentially to $c_{\min }$, where $c_{\min }$ represents the maximum insulation effect of char in the range of $[0,1]$.

6.1.4 Reaction Rate Coefficient. Finally, we assume that the mass loss rate is proportional to the surface temperature $T$. As we only define temperatures on the branch surface, we obtain the rate of change of mass from the branch surface temperature. We introduce a temperature dependent reaction rate $k(T)$ and define the rate of change of mass for virgin wood as an ordinary differential equation:

$$
\mathrm{d}_{t} M+k c A=0 \text {. }
$$

The rate of change of mass depends on the dimensionless char insulation parameter $c$, the area of the pyrolyzing front $A$, and the reaction rate given by

$$
k(T)=\Delta m \cdot \begin{cases}0 & T<T_{0} \\ S\left(\left(T-T_{0}\right) /\left(T_{1}-T_{0}\right)\right) & T_{0} \leq T \leq T_{1} \\ 1 & T>T_{1}\end{cases}
$$

in which $S(x)=3 x^{2}-2 x^{3}$ describes a sigmoid-like function interpolating smoothly from zero to one for temperatures between $T_{0}=150^{\circ} \mathrm{C}$ and $T_{1}=450^{\circ} \mathrm{C}$.

Unlike our definition of $k$, the mass loss can also be computed based on the reaction rate coefficient defined in the Arrhenius equation [Mcnaught and Wilkinson 1997]. However, we found that a first order Arrhenius reaction scheme did not suffice as approximation of wood pyrolysis in our case. For our model we require an unignited state of wood which is not supported by the Arrhenius equation. Our reaction rate coefficient $k$ describes the rate of mass change adequately for both, the unignited and ignited, states of wood. Moreover, it simplifies the mathematical description of our model, while still qualitatively capturing the effects of temperature on mass loss rate.

The boundary condition for Eq. (7) is given by the density of wood $\rho$ and the initial mass $M_{0}=A_{0} / 2 H_{0} \rho$ of the volumetric portion of wood associated with the surface elements.

6.1.5 Moisture and Smoke. One of the main parameters of wood combustion is moisture, as it affects the temperature changes on the branch surface. A piece of wood with high water content can prolong pyrolysis to the point of prohibiting ignition altogether. We represent moisture in our model explicitly by a separate parameter $W$, with values in the range of $[0,1]$. High values of $W$ denote a piece of wood with a high level of water content and a low value represents dry wood. In our model we assume that moisture affects the rate of change of surface temperatures via the coefficient

$$
b=(1-W) b_{\text {dry }}+W b_{\text {wet }},
$$

where $b_{\text {dry }}$ and $b_{\text {wet }}$ are coefficients representing the heat transfer rates for dry and wet wood. We calculate the value of the water content $W$ based on the evapotation rate $w$ and the surface temperature $T_{\mathrm{s}}$ by solving the ordinary differential equation:

$$
\mathrm{d}_{t} W+w T_{\mathrm{s}}=0 .
$$



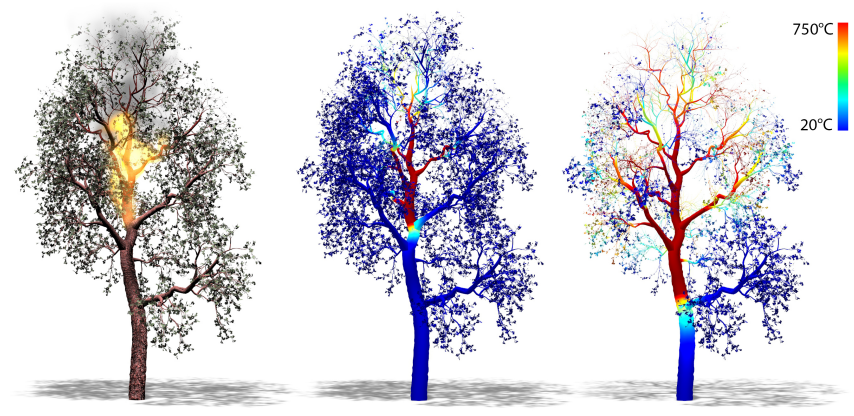

Fig. 5. The combustion in the branching structure (left) causes a bidirectional heat transfer in wood and leaves (middle, right). The color indicates the temperature variations on the surface from $20^{\circ} \mathrm{C}$ (blue) to $750^{\circ} \mathrm{C}$ (red).

Our fire simulation (Sec. 5) expresses smoke by the smoke density parameter $S$ (Eq. 3). We control the amount of smoke in our fire simulation by a combustion dependent source term $s_{S}$. Specifically, we assume that denser smoke is the result of wood combustion with higher moisture content $W$ :

$$
s_{S}=-S_{M} \mathrm{~d}_{t} M-S_{W} \mathrm{~d}_{t} W,
$$

where $S_{M}$ and $S_{W}$ are coefficients to express the rate at which smoke is added proportionally to the mass loss and the evaporation of water.

\subsection{Heat Transfer}

Combustion of wood is expressed in terms of bidirectional heat transfer between tree models and the surrounding air. A comparatively higher air temperature raises branch surface temperatures, which, when high enough, results in an increase of the mass loss rate (Eq. 7). The mass loss of surface elements, on the other hand, induces an increase in air temperature (Eq. 2, 13). Therefore, the heat transfer constitutes a positive feedback loop that enables the spread of flames in our model.

6.2.1 Surface Heat Transfer. The temperature $T_{\mathrm{S}}$ of a surface element is determined by taking into account the heat diffusion on the branch surface and the conduction of heat between air and surface. The heat conduction between air and branch surface (surface elements) is proportional to the difference in temperature between them. The rate of change of surface temperature $T_{\mathrm{S}}$ is thus given by

$$
\partial_{t} T_{\mathrm{s}}=a \nabla^{2} T_{\mathrm{s}}+b\left(T_{\mathrm{a}}-T_{\mathrm{s}}\right)
$$

The left-most term on the right side of the equation describes the heat diffusion on the branch surface using the Laplace operator and a temperature diffusion coefficient $a$. The right-most term expresses Newton's Law of Cooling. We assume that the heat transfer coefficient $b$ for surface elements can vary with different amounts of water content $W$, defined in Eq. (9). Fig. 5 shows the surface temperature on the branching structure of a tree model.

The radial heat transfer is not modeled explicitly by diffusion equations. Our model decouples the combustion in radial and tangential directions by introducing a pyrolyzing front for the radial direction and surface heat conduction for the tangential. Thus, the rate of combustion in those principal directions is controlled by separate Eqs. (7) and (12).
6.2.2 Air Heat Transfer. The heat transfer from branch surface to air is expressed by the surface heat transfer term $s_{\mathrm{T}}$ in Eq. (2). The surface heat transfer term expresses the combustion of flammable gases released by the process of pyrolysis. In our model this complex movement and combustion of gases is approximated by the mass loss $\mathrm{d}_{t} M$ determined in Eq. (7). Due to the combustion of flammable gases, the temperature increases proportionally to the mass loss:

$$
s_{\mathrm{T}}=-T_{\mathrm{M}} \mathrm{d}_{t} M,
$$

where $T_{\mathrm{M}}$ is a coefficient expressing the rate at which heat is added to the air by the combustion of gases released by the process of pyrolysis. We do not model the conduction of heat from wood tissue to air. The Laplace-Beltrami operator in Eq. (12) is discretized using the graph Laplacian. We use forward Euler integration for all differential equations in this section.

\subsection{Structural Deformation}

The pyrolysis of wood causes geometric deformations of the branching structure. We capture these effects by exploiting our particlebased tree representation (Sec. 4) and the associated surface mesh.

6.3.1 Branch Surface Deformation. Due to the contraction of char, the volume of wood decreases. We model this effect by deforming the surface mesh of the branches (Fig. 6). Vertices affected by pyrolysis are moved along their original normal direction, where the offset can be obtained by the thickness of the char layer $H_{\mathrm{c}}$, the remaining virgin wood $H$, and the original thickness of the branch $H_{0}$ :

$$
H_{\text {off }}=H_{0}-H-H_{\mathrm{c}} \text {. }
$$

The strength of the deformation is controlled by the contraction factor $\kappa$, which determines the amount the char layer contracts as a result of the pyrolysis (Eq. 5). Since $H_{\text {off }}$ is always less than $H_{0}$ (the char layer stops forming when the total mass of the element is pyrolyzed), a vertex never moves further than to its projection point on the branch, which guarantees that the mesh does not degenerate.

6.3.2 Tree Model Deformation. We compute the bending and breaking of branches by computing strains for twisting, stretching, bending, and shearing for each particle [Kugelstadt and Schömer 2016]. A branch breaks when the strain $\varepsilon$ exceeds a threshold $\varepsilon_{\max }$ $\left(\varepsilon>\varepsilon_{\max }\right)$. Then the rod element between the particles is removed and the physics of the detached particles are further simulated. To influence the dynamics of tree motions through the combustion process, we update the particles according to geometric changes of the model. Moreover, the mass loss caused by the combustion influences the stiffness of a branch, which causes it to bend and break more easily. Gravity is applied as an external force to all particles.

The mass of overhanging branches increases the strain, however, thicker branches are stiffer than thinner branches and therefore experience less strain. We approximate the maximum strain $\varepsilon_{\max }$ a branch can endure using the following equation:

$$
\varepsilon_{\max }=\frac{c_{d}}{d} \cdot\left(1-\frac{\sum M_{0}-\sum M}{\sum M_{0}}\right),
$$

where $d$ is the thickness of the branch at a particle, $M_{0}$ denotes the mass at the start of the simulation, $M$ the current mass of the surface 


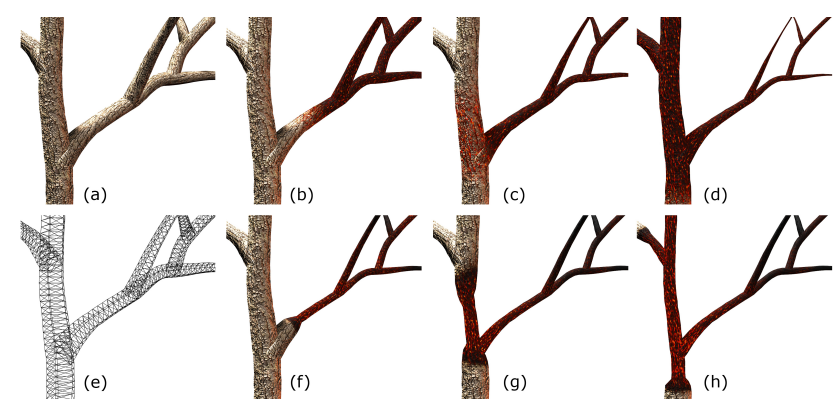

Fig. 6. We compute the combustion of branches (a) based on a uniform triangular surface mesh (e); after reaching the ignition temperature (b), the combustion causes deformations of the branches; the pyrolysis process (c) creates a charcoal layer on the branches (d). Compared to the simpler model ( $f-h)$, our combustion simulation allows to produce more realistic deformations of the branching structure.

elements associated with the particle, and $c_{d}$ a user defined coefficient. Branches that undergo combustion are penalized inversely proportional to their thickness to account for the effects of the mass of the overhanging sub-branches.

6.3.3 Leaves. Similar to branches, leaves burn individually and transfer heat to the surrounding environment. We represent leaves as two-dimensional surfaces with associated mass and area. Leaves are attached to branch particles (Sec. 7), thereby fires can spread from branches to leaves and vice versa. We simulate the mass loss based on Eq. (7) without the char insulation term and the heat diffusion based on Eq. (12). During the combustion process, the area of leaves decreases proportionally to their mass loss analogously to Eq. (14).

\section{IMPLEMENTATION AND RESULTS}

Our framework is implemented in C++ using OpenGL 4.2 and CUDA 8.0 on a desktop computer with an Intel Xenon CPU clocked at 3.7 Ghz and 32 GB of RAM. We did not specifically optimize our code and rendered all results shown in the paper and the accompanying video with a NVIDIA Titan X GPU in our framework.

7.0.1 Fire and Smoke. We simulate fire with a grid based fluid solver and render it with a GPU-based raymarching approach to capture the characteristic volumetric features of flames. To maintain interactive frame rates, we compute the involved grids for fire velocity, temperature, and smoke at different resolutions. For most of our renderings we used a fire velocity grid of size $64^{3}$ and grids of size $128^{3}$ to compute temperature and smoke. The size of the fluid domain is chosen to keep $d x$ small, but big enough to avoid effects from boundary conditions. For most of our experiments $d x$ is $5-10 \mathrm{~cm}$. We use a single time step of $30 \mathrm{~ms}$ per frame. The final color and opacity of the fire at a given voxel is determined by the temperature value and a transfer function [Nguyen et al. 2002]. We use a two pass Gaussian filter to approximate light scattering around the flames to add the impression of brightness and glow. Finally, we advect point sprites with the fire simulation to mimic the effect of sparks.

7.0.2 Branch and Leaf Geometry. We compute a triangular surface mesh for the branches of tree models based on the hierarchical rod structure defined in Sec. 4. For each chain of rod elements we compute a consecutive and uniformly tessellated mesh based on generalized cylinders. The start and end of a cylinder is defined by the radius and orientation of two subsequent particles. If more than one rod element starts at a particle, the rod element with the closest change of orientation is chosen to be the consecutive element to form a smooth branch, all other rod elements start a new branch with a corresponding mesh.

The surface heat transfer is directly computed on the tree mesh. It is important to note that the computed mesh is not a smooth manifold; meshes of child branches are not connected with the geometry of parent branches. We use an index buffer to store the adjacency information of mesh triangles to simulate heat diffusion. For vertices at the starting end of disconnected child branch meshes, adjacency information is not available. For these vertices, we select the closest vertex of the closest branch as the neighbor. The index buffer is computed as a preprocessing step.

7.0.3 Animation and Rendering. We use Linear Blend Skinning [Le and Deng 2012] to animate the tree mesh according to the positional changes of the branch particles. Each vertex is associated with the two particles of its corresponding rod element. The vertex is animated by computing its position updates based on the weights given by its associated particles. We further pronounce the geometry of branches with displacement mapping and define leaves as points that are rendered as textured quad surfaces in the geometry shader. To animate leaf motion, we associate each leaf with its closest branch particle; positional changes of branch particles cause updates of the corresponding leaf positions. Branch collisions are resolved by particle-particle collision tests through PBD.

We use texture blending on the branch mesh to animate the combustion process. A parameter for charring describes the visual degree of darkening of the surface, which is proportional to the thickness of the char layer. To animate glowing of the surface, we use a glow parameter that is set to 1 when the surface temperature of the surface reaches a threshold; it decays separately from the temperature to retain more control of the visualization.

\subsection{Performance and Parameters}

We measured the performance of our system for the figures shown in the paper; Tab. 1 shows timings and parameters of our system. All three components (dynamic tree motions, fire simulation, and combustion model) can be efficiently computed at interactive rates. Our method uses 9 user-controlled parameters that allow us to control the physical and biological properties of the combustion process. These parameters can be adjusted and explored interactively in our system. Additionally, we rely on seven physically-motivated parameters in our equations. These parameters are initialized once and remain constant across different simulations and tree models. Tab. 2 lists all parameters, their explanation, ranges, and units.

Our goal is to provide a mathematical model for wood combustion with a minimal set of parameters, while still allowing to model the key properties of burning trees, such as for pyrolysis, moisture, and charring. The introduced parameter space captures a diversity of realistic combustion patterns (Fig. 7, a). For example, the resistance of trees to fire is defined in two ways. First, by modulating the 
Table 1. Performance measures and parameter settings for figures in the paper. For fire, TF denotes the time for the simulation. For trees, NP is the number of particles, NV and NL represent the number of vertices and leaves, and TT is the time required to compute dynamic motions. The combustion model is computed based on the parameters listed in Tab. 2 and TC is the reported time for the computation. $(\dagger)$ indicates parameter ranges for multiple tree models that we did not explicitly capture.

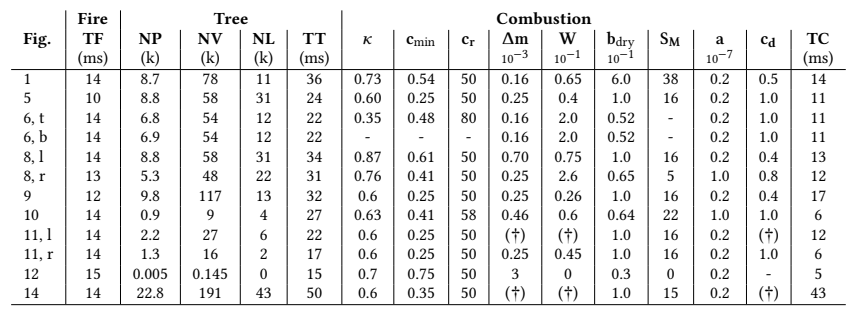

Table 2. Parameters used in our system. The upper half lists parameters that can be interactively controlled, whereas the bottom half shows parameters that remain constant for different simulations and tree models.

\begin{tabular}{l|l|l|l} 
Name & Description & Range & Units \\
\hline$\kappa$ & Char contraction factor & {$[0.5,1.0]$} & 1 \\
$c_{\min }$ & Min. value of c as a result of charring & {$[0.0,1.0]$} & 1 \\
$c_{r}$ & Rate of insulation due to charring & {$[50,250]$} & $1 \mathrm{~m}^{-1}$ \\
$\Delta m$ & Mass loss rate & {$[0.01,10.0] \times 10^{-3}$} & $1 \mathrm{~kg} \mathrm{~m}^{-2} \mathrm{~s}^{-1}$ \\
$W$ & Initial moisture (water content) & {$[0.0,1.0]$} & 1 \\
$b_{\mathrm{dry}}$ & Heat transfer coeff. for dry wood & {$[0.03,0.1]$} & $1 \mathrm{~s}^{-1}$ \\
$S_{M}$ & Amount of smoke from wood combustion & {$[0,400]$} & $1 \mathrm{~kg}^{-1}$ \\
$a$ & Temperature diffusion coeff. (wood) & {$[0.1,1.0] \times 10^{-7}$} & $1 \mathrm{~m}^{2} \mathrm{~s}^{-1}$ \\
$c_{\mathrm{d}}$ & Max. strain coeff. & {$[0.0,1.0]$} & $1 \mathrm{~m}^{-1}$ \\
\hline$\alpha$ & Temperature diffusion coeff. (air) & $0.8 \times 10^{-4}$ & $1 \mathrm{~m}^{2} \mathrm{~s}^{-1}$ \\
$\gamma$ & Radiative cooling coeff. & -0.008 & $1 \mathrm{~s}^{-1}$ \\
$\rho$ & Density of wood & 800 & $1 \mathrm{~kg} \mathrm{~m}^{-3}$ \\
$b_{\text {wet }}$ & Heat transfer coeff. for moist wood & $0.1 \cdot b_{\mathrm{dry}}$ & $1 \mathrm{~s}^{-1}$ \\
$w$ & Water evaporation rate & $0.3 \times 10^{-3}$ & $1 \mathrm{~s}^{-1} \mathrm{C}^{-1}$ \\
$S_{W}$ & Amount of smoke from water evaporation & 200 & 1 \\
$T_{M}$ & Amount of heat generated in combustion & $1.2 \times 10^{-6}$ & $1 \mathrm{C}^{\circ} \mathrm{kg}^{-1}$ \\
\hline
\end{tabular}

charring parameters, i.e. char contraction factor $\kappa$ and maximum insulation parameter $c_{\min }$ (Fig. 7, b), second, by changing the rate of mass loss using $\Delta m$ (Fig. 7, e). The first approach captures different wood charring dynamics, whereas the second allows to express wood combustion dynamics due to, e.g., the lack of wood resins such as in Redwood trees. Thus, a user has control over the rate and extent of flame spread. Further, our method exposes a parameter, called $\epsilon_{\max }$, to control the brittleness of branches as part of the wood combustion.

In Fig. 7 (c), a high value for the maximum strain $\epsilon_{\max }$ has been chosen, leading to frequent branch breaking events and a visibly damaged tree structure after the combustion. One of the most important specifiers of wood combustion in real trees is the water content as it majorly influences both, the flame spread as well as the amount and type of smoke produced. Our model takes this important phenomenon into account by controlling the smoke generation via the moisture parameter $W$, illustrated in Fig. 7 (d).

\subsection{Results}

The examples in Figs. 1 and 8 illustrate the capabilities of our framework. Different tree models are set on fire by igniting branches with a fire emitter. Due to the structure of the branches and individual settings for the parameters for each of the models, our

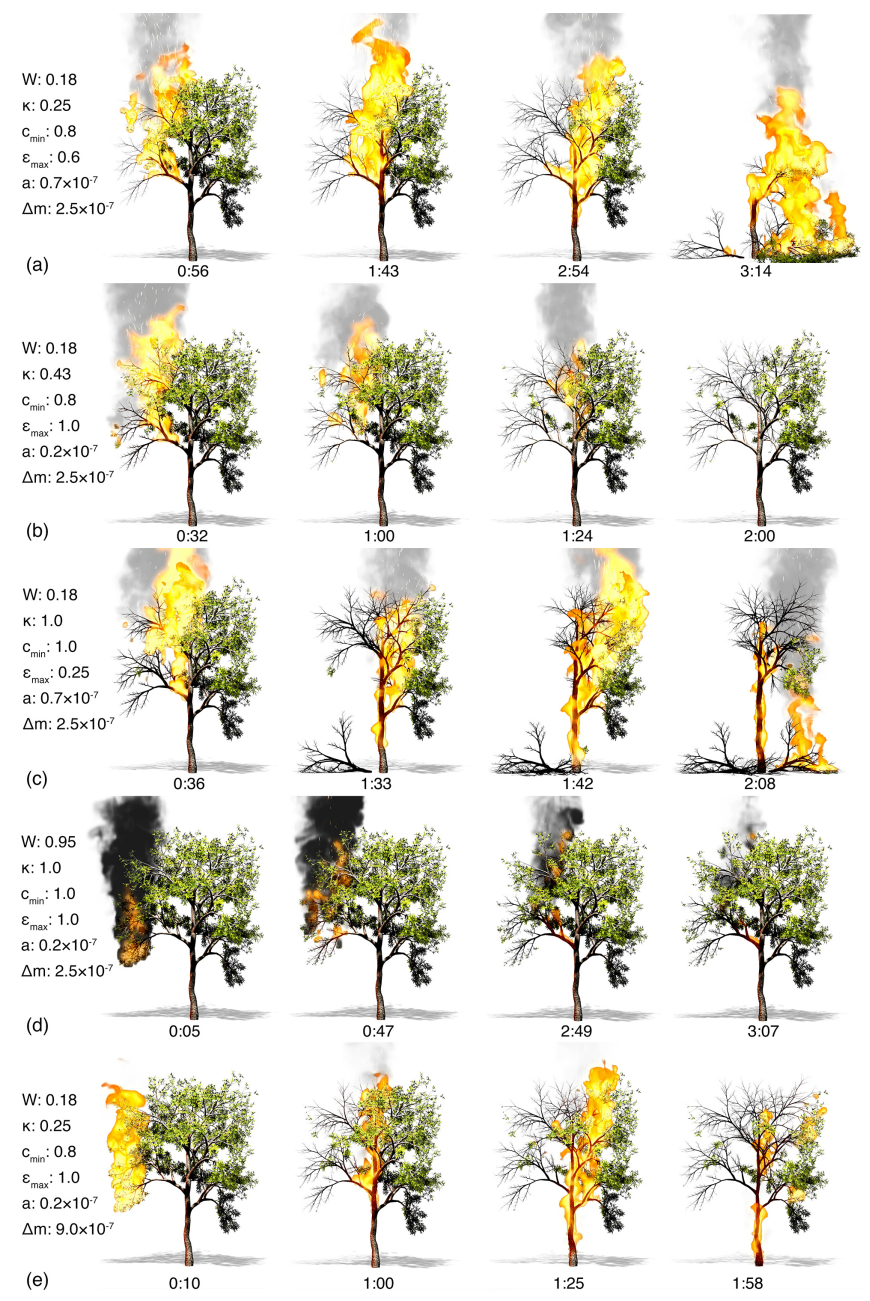

Fig. 7. Changing the parameters of our combustion simulation allows generating a variety of biologically- and physically plausible effects. The reported parameters can be adjusted in real-time to explore the parameter space.

method allows to plausibly simulate the combustion of botanical tree models (Fig. 8, a-e). The geometric features of the model affect the combustion process: the sparser branching structure of the deciduous tree model (Fig. 8, f-i) and higher moisture of its leaves hinder the fire to fully propagate through the model.

Fig. 8 and Fig. 9 show the interaction with burning tree models. The user can drag the branches of a model. They sway back and the flames propagate through the tree model. Dynamic motions of branches causes changes in the fire simulation and thereby affect the combustion of the model. Due to the combustion and externally induced stress, branches can bend and break, while they continue to burn. As the combustion progresses, the insulation of char hinders the combustion to proceed (Fig. 9, e, f).

In Fig. 10 we ignited the tree models in different locations. While the flames slowly propagate from the top towards the lower branches $(\mathrm{a}-\mathrm{c})$, the model rapidly catches fire when it is initially ignited at the bottom $(\mathrm{d}-\mathrm{g})$. We model heat transfer in wood and air, which allows fires to spread without the direct contact of flames and branches 

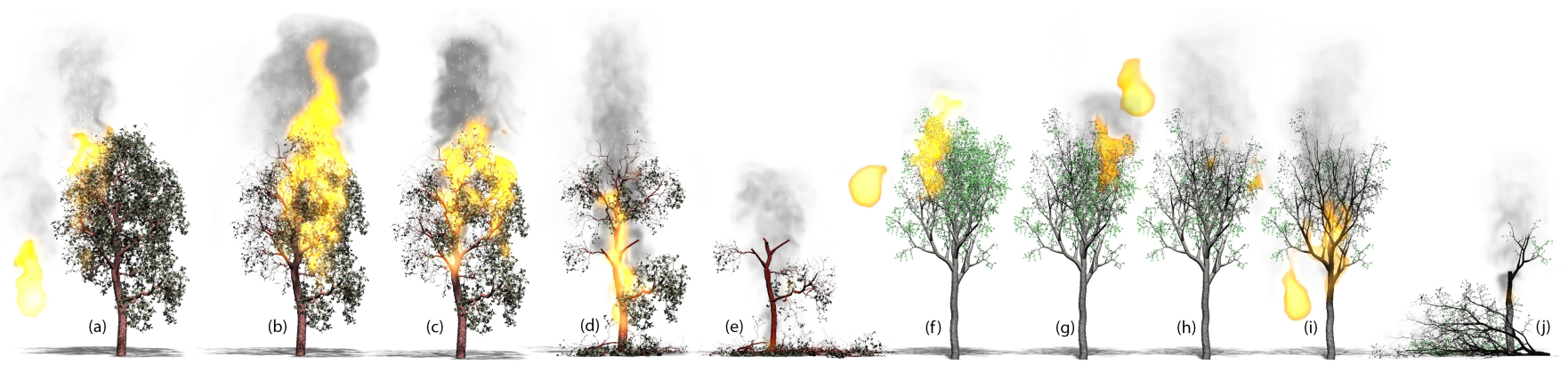

Fig. 8. A tree is set on fire (a) and flames propagate through the tree model (b, c). As the combustion progresses, branches start to decompose (d) until the insulation of char hinders the combustion to proceed (e). The geometric structure and different parameters affect the combustion process. Although ignited several times (f), (g) the tree does not catch fire. The flames quickly burn smaller branches and leaves $(\mathrm{h})$ that prohibit thicker branches to reach their ignition temperature. Only as the user ignites the bigger branches directly (i), they start to burn, which eventually causes the trunk to break (j).
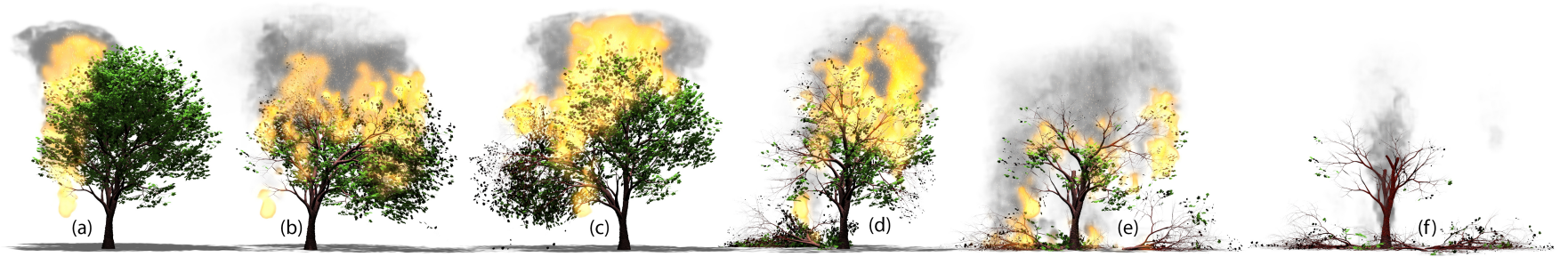

Fig. 9. The interaction with a burning tree model: the user sets a tree on fire (a) and interacts with the model by dragging its branches (b), (c). As a result of the combustion and the externally applied stress, branches start to break (d); the fire continues to burn on the detached branches (e) and eventually stops (f).
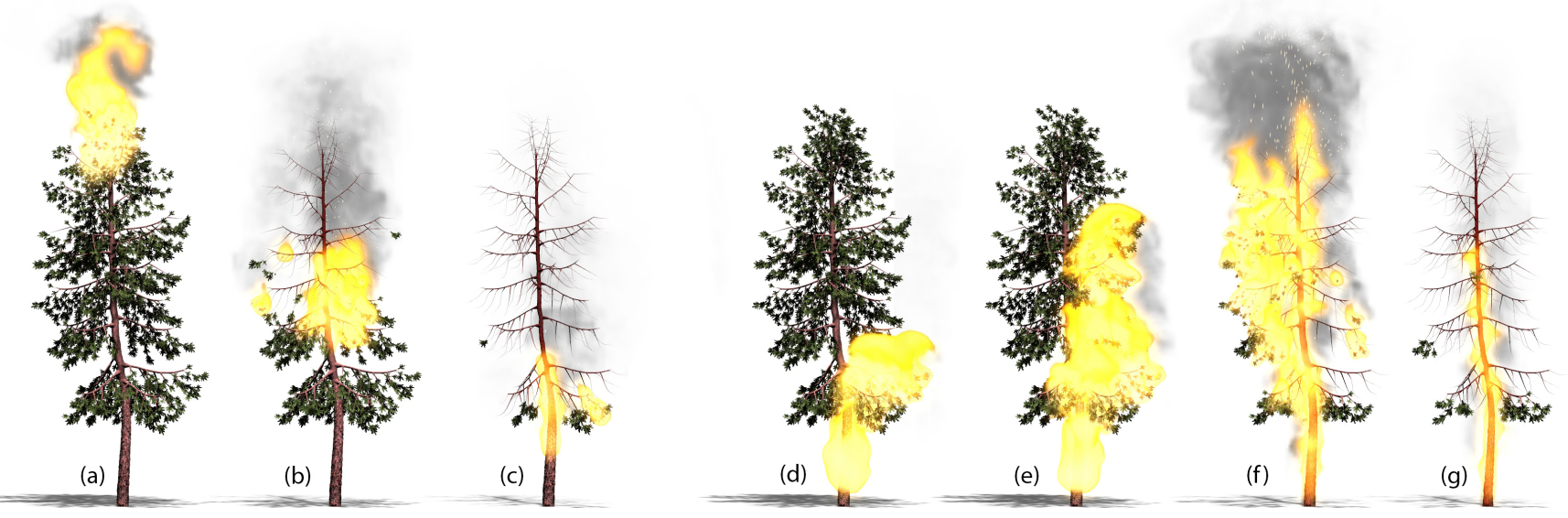

Fig. 10. The location of the initial flames causes different combustion behavior. The fir tree model on the left was ignited at the top (a) and heat transfer causes the model to slowly burn down to its trunk $(b, c)$. The model on the right was ignited at the bottom (d) and flames rapidly cover the entire model (e,f) until only the trunk is burning (g).
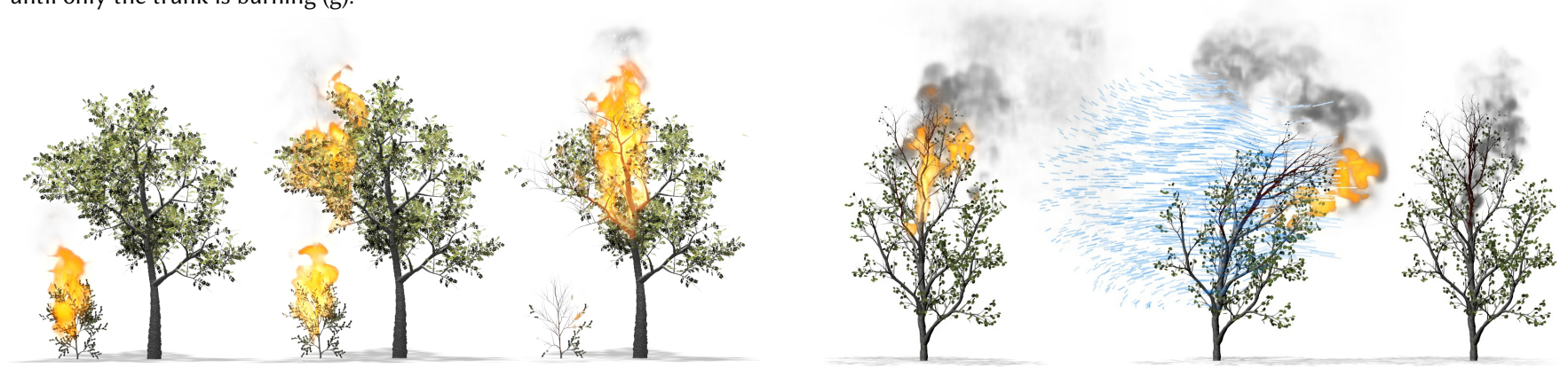

Fig. 11. Left: the flames of a burning bush progressively increase the surrounding air temperature until the branches of a tree reach their ignition temperature. Right: the user can interact with a burning tree by using a wind emitter to extinguish flames. 
(Fig. 11, left). Moreover, as we rely on a grid-based fluid solver it is possible to affect our fire simulation and the combustion model with wind. Fig. 11 (right) shows how the flames of a burning tree are extinguished by a wind gust.

Finally, Fig. 14 shows the combustion of a group of trees consisting of five models. A model is set on fire and starts to burn. The combustion propagates across the branching structure and to other models in the scene until all tree models are affected. The combustion slows down when no further fuel is available.

\section{EVALUATION, DISCUSSION, AND LIMITATIONS}

The pyrolysis of trees is a complex natural phenomenon that is difficult to observe and to sufficiently quantify. While many studies investigate the combustion of different tree species and the pyrolysis of woody materials in confined settings, such as in laboratories, there exists no dataset that provides a baseline for the combustion of geometric models of botanical trees.

\subsection{Evaluation}

To evaluate our computational model we provide visual and quantitative comparisons to experimental data of real wood combustion and compare our model with a simplified combustion model for branching structures.

8.1.1 Comparison to Real Wood Combustion. We conducted experiments where we burnt two wood samples fixated in a vertical and a skewed orientation. A flame was produced by a Bunsen burner at the lower ends of the sticks. Fig. 12 shows the setup of the experiment. We observed that pyrolysis progresses more rapidly when the sample is oriented vertically compared to when it is fixated in a skewed orientation. As the flames follow the inverse gravity direction, they quickly cover the entire stick, which causes a rapid pyrolysis of the wood (Fig. 12, top, left).

We quantify this observation by measuring the time of the combustion process, starting with the ignition of the material until no flame is visible anymore. An important quantitative measure of wood combustion in material sciences is the mass loss rate under a constant heat flux over time. Fig. 13 (left) shows that the mass loss is increasing very fast in the first few seconds of the experiment until reaching a well pronounced tipping point (red line). This tipping point coincides with the rapid formation of the char layer, which has an insulating effect for wood (Sec. 6.1.3) and contributes to a reduction of the mass loss rate [Weng and Fan 2007].

We compare these observations with our model by defining virtual wood samples in our particle-based representation (Sec. 4). The samples and a fire emitter are positioned in a scene to match the laboratory setup. We manually set up the parameters for this simulation to represent dry wood. Fig. 12 (bottom) shows that our model produces similar visual results for both scene setups. Please note, that our current model cannot handle the bending of wood samples due to more advanced effects such as the cracking of charcoal. Also, we do not simulate the release of resins responsible for the typical flickering of flames. The pyrolysis of the vertical sample took $87 \mathrm{sec}$. in the burning chamber and $81 \mathrm{sec}$. in our simulation. For the skewed sample we measured $165 \mathrm{sec}$. (burning chamber) and $235 \mathrm{sec}$. (simulation). Furthermore, the graph in Fig. 13 (left) shows

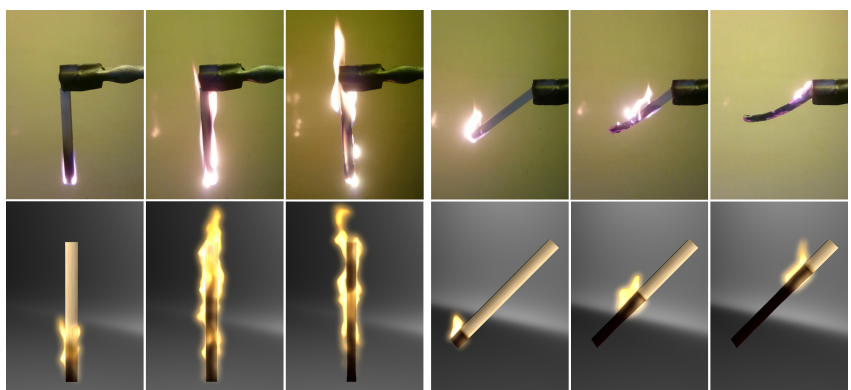

Fig. 12. Evaluation of the combustion model. We burned samples of wood in a burning chamber and simulated the pyrolysis with a similar setup with our method. Compared to the real pyrolysis, our method produces similar results for both sample arrangements: vertical (left), skewed (right).

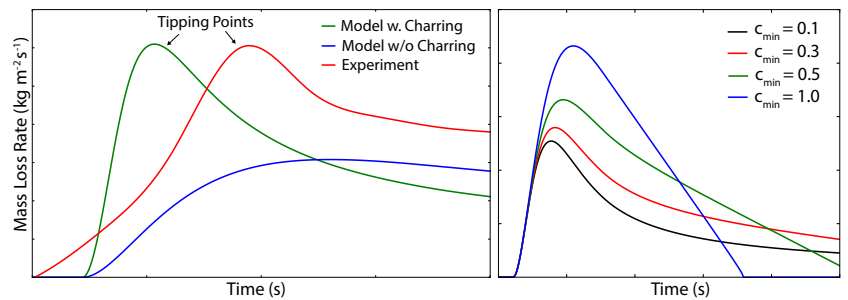

Fig. 13. Left: comparison of mass loss rates between experimental (vertical) and simulated data. Unlike the simpler model without charring (blue line), the model with charring simulation (green line) features a tipping point similar to the experimental dataset (red line). Right: Results for the mass loss rates with different values for $c_{\text {min }}$; low values indicate a high insulation.

that the mass loss curve in our model has similar characteristics compared to the mass loss curve of the real wood sample. The simulated mass loss curve (green line) expresses the exponential increase in the beginning, the tipping point, and the subsequent decrease in mass loss. A simpler model, without taking the effects of charring into account, but combusting at a similar rate (blue line), is missing the characteristic tipping point.

8.1.2 Simplified Model for Wood Combustion. Our mathematical model captures the essential phenomena in wood combustion, such as the formation of a pyrolyzing front and wood charring. It is necessary to capture these phenomena to obtain a realistic simulation of wood combustion. A naive model of solid combustion, where the insulating effects of charring are ignored and mass loss is proportional to the total mass of particles, yields unrealistic combustion patterns. In Fig. 6, we illustrate a comparison of a naïve approach with our method. In the simple model, thick and thin branch segments combust at similar rates, leading to unreasonable changes of branch thickness, whereas, in our approach thicker branches realistically combust proportionally much slower compared to thinner branches. Furthermore, in our model, the rate of mass loss can be controlled by the parameter $c_{\min }$ representing the insulation effect of a char layer. Fig. 13 (right) shows the mass loss rate as a result for different values of $c_{\text {min }}$, where low values indicate a high insulation and high values less insulation. 


\subsection{Discussion and Limitations}

Our focus was on defining a model to interactively explore combustion for botanical tree models. To compute this complex phenomenon in real-time, we carefully abstract and simplify components in our model. This includes the simulation of the temperature gradient inside the wood, the representation of char contraction, the fluid boundary conditions, and the explicit modeling of mass transfer from the branch surface to the surrounding air.

We do not use trees as a boundary condition for our fluid simulation, but instead use the heat and smoke generation terms $s_{T}$ (Eq. 2) and $s_{S}$, (Eq. 3), provided by our combustion model. However, including the tree geometry as a boundary condition would allow to more precisely model the convection of temperature currents around branches. A more accurate mass transfer would enable us to introduce a time delay between the heat transfer from air to surface and vice versa, which would allow a more realistic simulation of temperature changes on the branches. Moreover, we abstained from calculating an internal temperature gradient for branches (e.g. with a volumetric method) and instead express the movement of a pyrolyzing front associated with individual surface elements directly. Furthermore, we assume that the char contraction is constant for different types of wood. However, among other factors, the char contraction factor is known to be dependent on temperature and a more plausible hypothesis would yield more accurate results. Dynamic changes of the formation of charcoal could then be used to include the procedural formation of char cracking patterns or the bending of wood as part of our surface deformation approach.

Existing models for the combustion of trees and wood either focus on computationally evolved methods to analyze the physical processes of pyrolysis in detail [Thi et al. 2016] or neglected pyrolysis altogether to study the effects of wood combustion at a higher level of abstraction, e.g. for fire spread in forests [Seidl et al. 2012]. In material sciences, efforts are made to improve structural design by taking realistic fire scenarios into account. However, current methods, e.g. based on finite elements, are too slow to handle complex geometry. Here, real-time approaches can provide a way towards generating new insights. Methods that consider the tree geometry can significantly improve simulations for forest fire modeling.

\section{CONCLUSION AND FUTURE WORK}

We have presented a computationally efficient method for the combustion of botanical tree models. We simulate realistic fires and provide a novel method for the pyrolysis of wood, that allows us to express the mass loss of virgin wood, the insulating effect of char, and the temperature changes due to moisture evaporation. Our approach is based on a biologically-plausible mathematical formulation and supports modeling a variety of effects, including the deformation of lateral branch geometry, the bending and breaking of branches as well as the plausible animation of wood combustion. Our method is the first attempt to jointly simulate botanical tree models and wood combustion in a biologically plausible way. Thereby, our model can be viewed as a link between low- and highlevel methods with the potential to provide insights in a variety of fields, including material science and forestry research.
There are a number of open issues that appear as avenues for future work. It would be interesting to explore through further analysis, if our method can help to uncover how geometric, physical, and biological properties of trees contribute to fire spread across different plants. Moreover, we are currently approximating different phenomena, such as heat conduction on the surface and char contraction, as constant values in our mathematical framework. Increasing the complexity of these components would allow to improve the realism of combustion for botanical tree models.

\section{ACKNOWLEDGEMENTS}

The authors are grateful to Xiangmin Jiao and David E. Keyes for helpful discussions. Moreover, the reviewers' valuable comments that improved the manuscript are gratefully acknowledged. The work was supported by the NSF grant CCF-1514305, KAUST's baseline funding, and the Max Planck Center for Visual Computing and Communication (MPC-VCC) funded by Stanford University and the Federal Ministry of Education and Research of the Federal Republic of Germany (grants FKZ-01IMC01 and FKZ-01IM10001).

\section{REFERENCES}

Florence Bertails, Basile Audoly, Marie-Paule Cani, Bernard Querleux, Frédéric Leroy, and Jean-Luc Lévêque. 2006. Super-helices for Predicting the Dynamics of Natural Hair. Proc. of ACM SIGGRAPH (2006), 1180-1187. DOI : https://doi.org/10.1145/ 1179352.1142012

Craig F. Bohren and David B. Thorud. 1973. Two theoretical models of radiation heat transfer between forest trees and snowpacks. Agric. For. Meteorol. 11 (1973), 3 - 16. DOI : https://doi.org/10.1016/0002-1571(73)90047-2

Romain Casati and Florence Bertails-Descoubes. 2013. Super Space Clothoids. ACM Trans. Graph. 32, 4, Article 48 (2013), 12 pages. DOI : https://doi.org/10.1145/2461912. 2461962

Norishige Chiba, Kazunobu Muraoka, Hiromichi Takahashi, and Mamoru Miura. 1994 Two-dimensional visual simulation of flames, smoke and the spread of fire. FVCA 5, 1 (1994), 37-53. DOI : https://doi.org/10.1002/vis.4340050104

A. Demirbaş. 2000. Mechanisms of liquefaction and pyrolysis reactions of biomass ECM 41, 6 (2000), 633 - 646. DOI : https://doi.org/10.1016/S0196-8904(99)00130-2

Howard W. Emmons and Arvind Atreya. 1982. The science of wood combustion. Proceedings of the Indian Academy of Sciences Section C: Engineering Sciences 5, 4 (1982), 259. DOI : https://doi.org/10.1007/BF02904581

L. Hernández Encinas, S. Hoya White, A. Martín del Rey, and G. Rodríguez Sánchez. 2007. Modelling forest fire spread using hexagonal cellular automata. Appl. Math. Model. 31, 6 (2007), 1213 - 1227. DOI : https://doi.org/10.1016/j.apm.2006.04.001

Ronald Fedkiw, Jos Stam, and Henrik Wann Jensen. 2001. Visual Simulation of Smoke Proc. of ACM SIGGRAPH (2001), 15-22. DOI : https://doi.org/10.1145/383259.383260

Kathinka Leikanger Friquin. 2011. Material properties and external factors influencing the charring rate of solid wood and glue-laminated timber. Fire and Materials 35, 5 (2011), 303-327. DOI : https://doi.org/10.1002/fam.1055

A. Galgano and C. Di Blasi. 2005. Infinite- versus finite-rate kinetics in simpliefied models of wood pyrolysis. Combustion Science and Technology 177 (2005), 279-303.

Torsten Hädrich, Bedrich Benes, Oliver Deussen, and Sören Pirk. 2017. Interactive Modeling and Authoring of Climbing Plants. Eurographics (2017).

Jeong-Mo Hong, Tamar Shinar, and Ronald Fedkiw. 2007. Wrinkled Flames and Cellular Patterns. ACM Trans. Graph. 26, 3, Article 47 (2007). DOI : https://doi.org/10.1145/ 1276377.1276436

Yi Hong, Dengming Zhu, Xianjie Qiu, and Zhaoqi Wang. 2010. Geometry-based Control of Fire Simulation. Vis. Comput. 26, 9 (2010), 1217-1228. DOI : https://doi.org/10 1007/s00371-009-0403-8

Christopher Horvath and Willi Geiger. 2009. Directable, High-resolution Simulation of Fire on the GPU. ACM Trans. Graph. 28, 3, Article 41 (2009), 8 pages. DOI: https://doi.org/10.1145/1531326.1531347

Takashi Ijiri, Shigeru Owada, and Takeo Igarashi. 2006. Seamless Integration of Initial Sketching and Subsequent Detail Editing in Flower Modeling. Comp. Graph. Forum 25,3 (2006), 617-624.

T. Kugelstadt and E. Schömer. 2016. Position and Orientation Based Cosserat Rods. Proc of ACM SIGGRAPH/Eurographics SCA (2016), 169-178. http://dl.acm.org/citation. cfm?id=2982818.2982842

Michael J. Lawes, Anna Richards, Josefine Dathe, and Jeremy J. Midgley. 2011. Bark thickness determines fire resistance of selected tree species from fire-prone tropical 

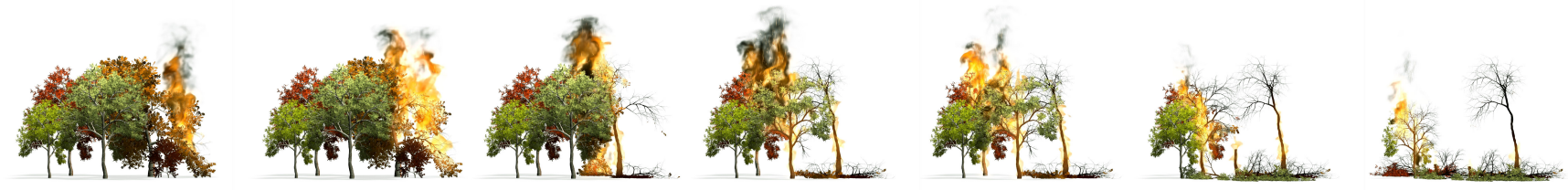

Fig. 14. The combustion of a complex scene of five tree models. From left to right: one of the models is set on fire and starts to burn; the combustion propagates across the branching structure and to other models, until all tree models are burning. The combustion slows down when no further resources are available.

savanna in north Australia. Plant Ecol. 212, 12 (2011), 2057-2069. DOI : https://doi. org/10.1007/s11258-011-9954-7

Binh Huy Le and Zhigang Deng. 2012. Smooth Skinning Decomposition with Rigid Bones. ACM Trans. Graph. 31, 6, Article 199 (2012), 10 pages. DOI : https://doi.org/ $10.1145 / 2366145.2366218$

Shiguang Liu, Tai An, Zheng Gong, and Ichiro Hagiwara. 2012. Physically Based Simulation of Solid Objects Burning. Springer Berlin Heidelberg, Berlin, Heidelberg, 110-120. DOI : https://doi.org/10.1007/978-3-642-29050-3_10

Yotam Livny, Soeren Pirk, Zhanglin Cheng, Feilong Yan, Oliver Deussen, Daniel CohenOr, and Baoquan Chen. 2011. Texture-lobes for Tree Modelling. ACM Trans. Graph. 30, 4, Article 53 (2011), 10 pages. DOI : https://doi.org/10.1145/2010324.1964948

Yang Lizhong, Chen Xiaojun, Zhou Xiaodong, and Fan Weicheng. 2002. A modified model of pyrolysis for charring materials in fire. Int. F. Eng. Sci. 40, 9 (2002), $1011-$ 1021. DOI : https://doi.org/10.1016/S0020-7225(02)00002-2

Steven Longay, Adam Runions, Frédéric Boudon, and Przemyslaw Prusinkiewicz. 2012. TreeSketch: interactive procedural modeling of trees on a tablet. In Proc. of the Intl. Symp. on SBIM. 107-120.

Robert William MacCormack and American Institute of Aeronautics. 1969. The effect of viscosity in hypervelocity impact cratering. Technical Report AIAA-69-354. Astronautics. http://opac.inria.fr/record=b1070875

A. D. Mcnaught and A. Wilkinson. 1997. IUPAC. Compendium of Chemical Terminology, 2nd ed. (the "Gold Book"). WileyBlackwell.

Z. Melek and J. Keyser. 2002. Interactive simulation of fire. Pacific Graphics (2002), 431-432. DOI : https://doi.org/10.1109/PCCGA.2002.1167889

Zeki Melek and John Keyser. 2005. Multi-representation Interaction for Physically Based Modeling. Proc. of SPM (2005), 187-196. DOI : https://doi.org/10.1145/1060244 1060265

Dominik L. Michels, J. Paul T. Mueller, and Gerrit A. Sobottka. 2015. A Physically Based Approach to the Accurate Simulation of Stiff Fibers and Stiff Fiber Meshes. Comput. \& Graph. 53B (2015), 136-146.

Matthias Müller, Bruno Heidelberger, Marcus Hennix, and John Ratcliff. 2007. Position Based Dynamics. F. Vis. Comun. Image Represent. 18, 2 (2007), 109-118. DOI : https: //doi.org/10.1016/j.jvcir.2007.01.005

Radomír Měch and Przemyslaw Prusinkiewicz. 1996. Visual models of plants interacting with their environment. In Proc. of SIGGRAPH. ACM, 397-410. DOI : https://doi.org $10.1145 / 237170.237279$

Boris Neubert, Thomas Franken, and Oliver Deussen. 2007. Approximate Image-based Tree-modeling Using Particle Flows. ACM Trans. Graph. 26, 3, Article 88 (2007) DOI : https://doi.org/10.1145/1276377.1276487

Duc Quang Nguyen, Ronald Fedkiw, and Henrik Wann Jensen. 2002. Physically Based Modeling and Animation of Fire. ACM Trans. Graph. 21, 3 (2002), 721-728. DOI : https://doi.org/10.1145/566654.566643

Makoto Okabe, Shigeru Owada, and Takeo Igarashi. 2007. Interactive Design of Botanical Trees Using Freehand Sketches and Example-based Editing. In ACM SIGGRAPH Courses. ACM, Article 26. DOI : https://doi.org/10.1145/1281500.1281537

Peter E. Oppenheimer. 1986. Real time design and animation of fractal plants and trees Proc. of SIGGRAPH 20, 4 (1986), 55-64. DOI : https://doi.org/10.1145/15886.15892

Dinesh K. Pai. 2002. STRANDS: Interactive Simulation of Thin Solids using Cosserat Models. Comp. Graph. Forum 21, 3 (2002), 347-352. DOI : https://doi.org/10.1111/ 1467-8659.00594

Wojciech Palubicki, Kipp Horel, Steven Longay, Adam Runions, Brendan Lane, Radomír Měch, and Przemyslaw Prusinkiewicz. 2009. Self-organizing Tree Models for Image Synthesis. ACM Trans. Graph. 28, 3, Article 58 (2009), 10 pages. DOI : https://doi.org/ $10.1145 / 1531326.1531364$

W.J. Parker. 1989. Prediction Of The Heat Release Rate Of Douglas Fir. Fire Saf. Sci. 2 (1989), 337-346.

Vincent Pegoraro and Steven G. Parker. 2006. Physically-based Realistic Fire Rendering EG Nat. Phenom. (2006), 51-59. DOI : https://doi.org/10.2312/NPH/NPH06/051-059

Sören Pirk, Till Niese, Oliver Deussen, and Boris Neubert. 2012. Capturing and animating the morphogenesis of polygonal tree models. ACM Trans. Graph. 31, 6, Article 169 (2012), 10 pages. DOI : https://doi.org/10.1145/2366145.2366188

Sören Pirk, Till Niese, Torsten Hädrich, Bedrich Benes, and Oliver Deussen. 2014. Windy Trees: Computing Stress Response for Developmental Tree Models. ACM Trans.
Graph. 33, 6, Article 204 (2014), 11 pages. DOI: https://doi.org/10.1145/2661229. 2661252

Sören Pirk, Ondrej Stava, Julian Kratt, Michel Abdul Massih Said, Boris Neubert, Radomír Měch, Bedrich Benes, and Oliver Deussen. 2012. Plastic trees: interactive self-adapting botanical tree models. ACM Trans. Graph. 31, 4, Article 50 (2012), 10 pages. DOI : https://doi.org/10.1145/2185520.2185546

P. Prusinkiewicz. 1986. Graphical applications of L-systems. In Proc. on Graph. Interf. 247-253.

Rupert Seidl, Werner Rammer, Robert M. Scheller, and Thomas A. Spies. 2012. An individual-based process model to simulate landscape-scale forest ecosystem dynamics. Ecological Modelling 231 (2012), 87 - 100. DOI : https://doi.org/10.1016/j. ecolmodel.2012.02.015

Andrew Selle, Michael Lentine, and Ronald Fedkiw. 2008. A mass spring model for hair simulation. ACM Trans. Graph. 27, 3, Article 64 (2008), 11 pages. DOI : https: //doi.org/10.1145/1360612.1360663

Jos Stam. 1999. Stable Fluids. Proc. of ACM SIGGRAPH (1999), 121-128. DOI : https: //doi.org/10.1145/311535.311548

O. Stava, S. Pirk, J. Kratt, B. Chen, R. Měch, O. Deussen, and B. Benes. 2014. Inverse Procedural Modelling of Trees. Comp. Graph. Forum 33, 6 (2014), 118-131. DOI : https://doi.org/10.1111/cgf.12282

Alexey Stomakhin, Craig Schroeder, Chenfanfu Jiang, Lawrence Chai, Joseph Teran, and Andrew Selle. 2014. Augmented MPM for Phase-change and Varied Materials. ACM Trans. Graph. 33, 4, Article 138 (2014), 11 pages. DOI : https://doi.org/10.1145/ 2601097.2601176

V.D. Thi, M. Khelifa, M. El Ganaoui, and Y. Rogaume. 2016. Finite element modelling of the pyrolysis of wet wood subjected to fire. Fire Safety fournal 81 (2016), 85 - 96 DOI : https://doi.org/10.1016/j.firesaf.2016.02.001

Bohan Wang, Yili Zhao, and Jernej Barbič. 2017. Botanical Materials Based on Biomechanics. ACM Trans. on Graphics (SIGGRAPH 2017) 36, 4 (2017)

W.G. Weng and W. C. Fan. 2007. A pyrolysis model of charring materials considering the effect of ambient oxygen concentration. Fire and Materials 31 (2007), 463-475.

Yili Zhao and Jernej Barbič. 2013. Interactive Authoring of Simulation-ready Plants. ACM Trans. Graph. 32, 4, Article 84 (2013), 12 pages. DOI : https://doi.org/10.1145/ 2461912.2461961

Ye Zhao, Xiaoming Wei, Zhe Fan, A. Kaufman, and Hong Qin. 2003. Voxels on fire [computer animation]. In IEEE Visualization, 2003. VIS 2003. 271-278. DOI : https: //doi.org/10.1109/VISUAL.2003.1250382 